\title{
XXIV.
}

\section{Ueber die Betheiligung der Zellschicht des Chorion an der Bildung der Serotina und Reflexa.}

(Aus dem pathologisch-anatomiscben Institut in Bern.)

\author{
Von Anna Reinstein-Mogilowa \\ aus Skwira, Kiew'schen Gouvernements.
}

(Hierzu Taf. IX.)

Die folgende Arbeit beschäftigt sich mit den Ergebnissen, welche ich durch Untersuchung einiger junger Eier aus dem ersten bis zweiten Monate gewonnen habe. Sie betreffen vorzugsweise die Frage, inwiefern bei der Bildung der sogenannten Decidua fötale Elemente betheiligt sind.

Seitdem die erste genaue mikroskopische Untersuchung die Zusammensetzung der Decidua aus Zellen nachgewiesen hatte, wurden bis vor wenigen Jahren ganz unbestritten nicht blos die Vera, sondern auch die Reflexa und Serotina als mütterlichen Ursprungs angesehen. Sie stellten die veränderte Mucosa uteri dar. Erst in den letzten Jahren erhoben sich $Z$ weifel, ob nicht ein Theil des serotinalen Gewebes fötalen Ursprungs sei. Es entstanden diese Zweifel in Anschluss an die Beobachtungen, welche Langhans ${ }^{1}$ ) ïber die sogenaunte Zellschicht des Chorion mittheilte. Es lieferte derselbe den Nachweis, dass Dasjenige, was man früher als Chorionepithel bezeichnete, in der frühesten Periode doppeltschichtig ist: Eine obere Lage von dunkelkörnigem Protoplasma ohne Zellgrenzen, mit sehr zahlreichen kleinen Kernen liegt auf einer tieferen Lage, welche aus einer Schicht scharf gegen einander abgegrenzter, grosser, bald platter, bald würfelförmiger, selbst manchmal cylindrischer Zellen besteht. Der oberen, welche den älteren Schilderungen des Chorionepithels zu Grunde liegt, lässt er diesen Namen. Die

1) Beiträge zur Anatomie und Embryologie. 1882. S. 69. 

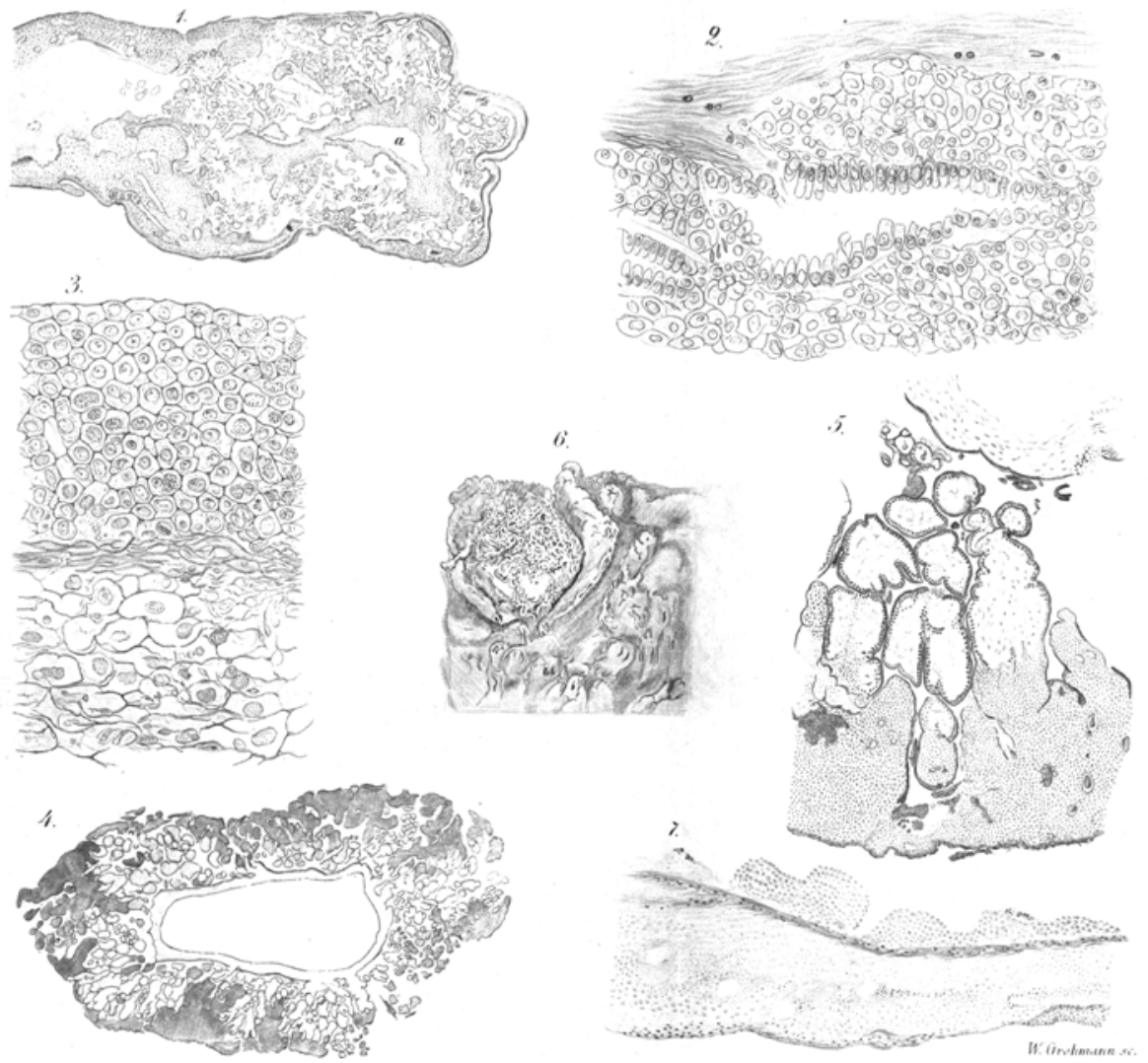
untere bezeichnete er als Zellschicht. Beide Lagen sind sehr eng mit einander verbunden und jede derselben erscheint von den ersten Stadien an, die er beobachten konnte, als continuirliche, besondere Membran. Das Chorionepithel sieht er, nach den damals allgemein angenommenen Anschauungen, als epithelialer Theil der serösen Hülle an; in der tieferen vermuthet er die lang gesuchte mesodermale Lage der gleichen Membran.

Die Zellschicht spielt nach seiner Auschauung in der Bildung der Placenta eine sehr wichtige Rolle. Sie hat nehmlich die Function, die erste Verbindung zwischen fötalen und mütterlichen Eihäuten herzustellen. Ueberall, wo Zotten und Chorion mit der Mucosa uteri in Verbindung treten, wuchert die Zellschicht und tritt in engste Verbindung mit dem ähnlich gebauten mütterlichen Gewebe. Sie bildet auch ferner eigenthümliche insuläre grosse Zellknoten, welche an den Zottenspitzen zwischen den Zotten und an dem Chorion sich finden, - Knoten, die er selbst früher ${ }^{1}$ ) genau beschrieben und als mitterlichen Ursprungs angesehen hatte.

Diese Zellschicht hat bis jetzt noch keine allgemeine Anerkennung gefunden. Nur Kastschenko ${ }^{2}$ ), Minot ${ }^{3}$ ) and Keibe $1^{4}$ ) bestätigen ihre Existenz. Von diesen Autoren beschäftigt sich Kastschenko am ausführlichsten mit ihr. Er weicht in manchen Beziehungen von J anghans ab. Zunächst hinsichtlich der Beziehung zu dem bedeckenden Chorionepithel. Nach ihm sondern sich aus dem letzteren die Elemente der Zellschicht allmählich aus, - ein Prozess, der sich noch in ziemlich späten Perioden vollzieht. Namentlich entstehen auf diese Weise die oben erwähnten insulären grosszelligen Knoten; weiter aber bildet sich aus diesen Zellknoten auch ein Theil der Serotina, ja die eigentlichen grossen Serotinazellen sind nach ihm nichts anderes, als Producte der Zellschicht, als Abkömmlinge des Chorionepithels. Er führt für diese Anschaung die Identität der Form der Zellen und zweitens die Thatsache an, dass die Kno-

1) Archiv f. Anatomie und Physiologie. 1877. S. 189.

$\left.{ }^{2}\right)$ Arcbiv f. Anatomie und Physiologie. 1885. S. 451.

3) Uterus and Embryo. The Journal of Morphology. 3 April 1889. I Rabbit. II man.

4) Anatomischer Anzeiger. September 1889. No. 17. S. 534. 
ten unabhängig von der Serotina entstehen und nachher, jedenfalls zum Theil, mit ihr verwachsen. „Untersuchen wir einen vertical durch das, in Zusammenhang mit der Mucosa serotina stehende, Chorion am Ende des ersten Monates geführten Schnitt, so finden wir zwischen den Zotten nur isolirte Zellknoten von unbedeutender Grösse. Je näher wir zu der Mucosa serotina rücken, desto grösseren Knoten begegnen wir, unter denen man oft solche findet, die offenbar aus mehreren kleinen, mit einander nur durch enge Balken zusammenhängenden Zellknoten zusammengesetzt sind. Daneben findet man auch grössere, einfache Knoten, über deren complicirten Ursprung man nur aus der grösseren Zahl der sie bildenden Zotten schliessen kann. Nähern wir uns noch mehr der Mucosa serotina, so finden wir auch solche Knoten, die, obwohl ganz den vorhergehenden ähnlich, doch nicht mehr frei liegen, sondern mit der Mucosa serotina verwachsen sind und eine Art Auswüchse oder Vorsprünge dieser Membran, welche sich zwischen die Zotten einsenken und zugleich die Endäste derselben einschliessen, bilden. Endlich bietet die ganze innere (dem $\mathrm{Ei}$ zugewandte) Fläche der Mucosa serotina eine continuirliche Schicht von demselben Gewebe, aus dem die Zellknoten bestehen" (S. 473-474).

Weiter finden wir in der Arbeit von Nitabuch ${ }^{1}$ ) für eine etwas spätere Periode dieselbe Idee ausgesprochen. Es beschreibt dieselbe eine eigenthümliche Schichtung an der Serotina: eine untere hellere, weniger gefärbte Schicht, mit grösseren länglichen fast spindelförmigen Zellen, die der Oberfläche parallel laufen, mit Riesenzellen in der Tiefe, Blutgefässen und Drüsen, darüber eine dünne Lage, welche sie als Fibrinstreif bezeichnet, und weiter eine ganz oberflächlich gelegene, vielfach unterbrochene Schicht, mit cubischen oder senkrecht gestellten Zellen, in welcher ausschliesslich eingewachsene Zotten liegen. Es wurde dabei die Vermuthung geäussert, dass die Fibrinstreifen die Grenze zwischen mütterlichem und fötalem Gewebe markiren, dass nur die darunter gelegene Schicht mütterlichen, die darüber gelegene aber - fötalen Ursprungs sei.

1) Nitabucb, Beiträge zur Kenntniss der menschlichen Placenta. 1887. Bern. Dissert. 
Meine Beobachtungen beziehen sich mehr auf die Frage, ob und inwiefern die Zellschicht sich an der Bildung der Serotina betheiligt. Die Differenzen zwischen Langhans und Kastschenko über das frühere Verhältniss dieser Schicht, ihre Beziehungen zum Chorionepithel - lasse ich im Wesentlichen unberührt. Ebenso auch die Frage, wie die beiden Schichten in embryologischer Beziehung aufzufassen sind. Eine Reihe von Embryologen hat sich in den letzten Jahren dem Studium des Baues und der Entwickelung der thierischen Placenta zugeweiht und es ist zu hoffen, dass gerade diese Fragen binnen Kurzem an einem vorwurfsfreien Material eine sichere Antwort erhalten. Ich gehe zur Schilderung meiner Beobachtungen über und stelle das $\mathrm{Ei}$ in den Vordergrund, von welchem mir von seiner weit grösseren Hälfte »wei ununterbrochene Schnittreihen zur Verfügung stehen.

I.

Das Ei stamint aus der Praxis des Herrn Privatdocenten Dr. Conrad. Fs rührt von einer sonst gesunden jungen Frau her, welche am 14. März 1889 sich verheirathet hatte. Die letzten Menses traten am 20. März ein. Wegen Fibroiden des Uterus wurde der künstliche Abortus durch Sondirung eingeleitet; er erfolgte am 16. Mai Vormittags. Das Li wurde sofort in Sublimat 1: 1000 gelegt; von Nachmittag an wurde es in feuchter Sublimatwatte aufbewahrt. Es wurde am 17. Mai Vormittags dem Herrn Prof. Langhans übergeben.

Das Ei, auf allen Seiten, wie es scheint, von Decidua umgeben, so dass von aussen Chorion oder dessen Zotten nicht sichtbar sind, ist von länglicher Form, etwas zapfenförmig, seine Länge misst $3 \frac{l}{2} \mathrm{~cm}$. Die queren Durchmesser sind etwas wecbselnd, da das Ei etwas collabirt erscheint. Auf einer Unterlage aufliegend, hat es eine Breite von $2 \frac{1}{2} \mathrm{crn}$, eine Dicke von $12-1 j \mathrm{~mm}$. Die Eihöhle wurde sofort eröffnet. Das Fruchtwasser ist klar. Wegen der Schlaffheit der Wände ist der Durchmesser der Eihöble nicht ganz sicher festzustellen; nur ungefähr lässt er sich auf $2 \frac{1}{2} \mathrm{~cm}$ berechnen. Es enthält einen gut entwickelten Embryo, dessen Länge vom Scheitel bis zum Schwanzende $18 \mathrm{~mm}$ beträgt, ohne Berücksichtigung der Krümmungen. Er erscheint etwas schlaff, aber seine äussere Form ist ganz normal. Die kurze, dicke Nabelsehnur ist leicht röthlich, gallertig.

Das Ei wird mit dem Embryo am 17. Mai Morgens 11 Ubr in 1 Sublimat und 9 Alkohol gelegt, kommt am 20. Mai in reinen Alcohol absolutus, der in den folgenden Tagen mebrfach erneuert wird; am 16. Juni wird es der Länge nach in zwei Hälften getrennt und zum $Z$ weck der Ganzfärbung für einige Tage in Alauncarmin gelegt, nachher nach tüchtigem Auswaschen 
und nochmaligem Einlegen in Alkobol in gewöhnlicher Weise in Celloidin eingebettet.

Ls wurde der grösste Theil desselben von mir in eine zusammenhängende Reihe von Schnitten mit dem Thoma'schen Mikrotom zerlegt. Zur Aufhellung wurde 0l. orig. angewandt, um die Auflösung des Celloidin zu vermeiden. Die Conservirung erfolgte in Balsam. So erbielt ich zwei Reihen von Schnitten, - eine Reihe von 190 und eine zweite von 132 Schnitten. Die Scbnitte hatten eine gleichmässige Dicke von $0,03 \mathrm{~mm}$.

Ich lege der übersichtlichen Schilderung der Verhältnisse einen Schnitt zu Grunde, welcher durch die Mitte des Eies gefallen ist, wo es also in seinem grössten Durchmesser getroffen ist (Fig. 1). Es hat hier im Allgemeinen eine länglich ovale Gestalt; dabei zeigt die äussere Contour, in Folge des Collabirens bei der Eröffnung, sowie der Schrumpfung in Alkohol, leichte Faltungen. Schon mit blossem Auge lassen sich zwei Abtheilungen erkennen. Die grösste, welche etwas mehr, als zwei Drittel des ganzen Schnittes einnimmt, entspricht dem gut erhaltenen Theil des Eies. Sie ist nach drei Seiten hin, und zwar an beiden langen Seiten, sowie an der einen schmalen, von einem continuirlichen schmalen Band von decidualem Gewebe umgeben. In der Mitte liegt das stark collabirte Chorion; dann folgen nach aussen die Zotten; dann, wie gesagt, die Decidua. Auch nach der anderen schmalen Seite (in der Abbildung links) schliesst sich zunächst an das Chorion eine breite Lage von Zotten an, auf welche die Serotina folgt, aber die letztere schliesst hier das Ei nicht ohne Weiteres ab, sondern die Bänder der Decidua, welche an den Längsseiten das Ei nach aussen begrenzen, laufen in gleicher Richtung, nur um Weniges genähert, weiter; der zwischen ihnen befindliche Raum enthält nach dem Ei hin einige Zottendurchschnitte, ist aber wesentlich vom Blut ausgefüllt; schliesslich enden die beiden Bänder frei, quer durchrissen. Das ist die einzige Stelle, an welcher das nach aussen abschliessende mütterliche Gewebe eine Unterbrechung erleidet. So entsteht die zweite Abtheilung des ganzen, Eies, die nur einen Anhang an die grössere bildet. Die Dimensionen der beiden Abtheilungen sind folgende: die grösste Abtheilung hat eine ziemlich gleichmässige Breite von $1 \mathrm{~cm}$, eine Länge von $2 \mathrm{~cm}$; die zweite, welche in Form eines dicken Zapfens an die eine schmale Seite der ersten sich anschliesst 
und sofort durch die gelbe Farbe des Blutes auffällt, ist $1 \mathrm{~cm}$ lang und 7-8 $\mathrm{mm}$ breit.

Das Chorion ist, wie gesagt, stark gefaltet, die von ihm umgebene eigentliche Eihöhle bildet eine sehr schmale Spalte, mannichfach verzogen; in ihr ist noch in vielen Schnitten das Amnion sichtbar. Der grösste Theil des Durchschnittes ist von dem grossen Raume eingenommen, der zwischen Serotina und Chorion sich findet. Ich will ihn in Folgendem mit dem, für die späteren Stadien in Gebrauch gekommenen, Namen des intervillösen Raumes bezeichnen.

Dies ist in der That ein einziger grosser Raum, ohne dass irgendwo vollständige oder unvollständige Scheidewände auch nur eine Andeutung davon geben, dass er durch Zusammenfliessen von kleinen Hohlräumen entstanden wäre. In ihm liegen die Zotten und noch einige andere Bestandtheile, auf die wir später zu sprechen kommen.

Was nun seine Ausdehnung anlangt, so umgiebt derselbe das Chorion von allen Seiten, nirgends steht letzteres mit der Decidua in directer Berührung. Es ist also der Unterschied zwischen Chorion villosum und Chorion laeve noch nicht ausgesprochen, aber doch wenigstens angedeutet durch die verschiedene Breite des fraglichen Raumes, sowie durch dje Zahl und Grösse der darin gelegenen Zotten. An der einen langen Seite beträgt nehmlich seine Breite etwa $3 \mathrm{~mm}$, dagegen an der gegenüberliegenden $-5-6 \mathrm{~mm}$, dabei sind an der letzteren Stelle die Zutten durchschnittlich viel dicker und in grösserer Zahl vorhanden; ganz besonders aber fällt die grosse Zahl von sehr breiten Zotten auf, wie sie an der anderen Seite sich gar nicht finden. An der einen schmalen durch die Decidua vollständig geschlossenen Seite gehen der schmale und breite Theil des intervillösen Raumes allmählich in einander über. An der anderen schmalen Seite dagegen ist der intervillöse Raum noch breit und enthält eine grosse Zahl von Zotten, die allerdings nicht auffallend dick sind.

Diese Verhältnisse, die verschiedene Breite des intervillösen Raumes, die Verschiedenheit in Zahl und Breite der darin enthaltenes Zotten, sind vorläufig die einzigen Zeichen dafür, dass die eine Hälfte des Chorions das Chorion frondosum oder villo- 
sum, die andere Seite das Chorion laeve darstellen dürfte. Es wäre also die dem ersteren gegenüber liegende Decidua als Serotina, die der letzteren gegenüber liegende als Reflexa zu bezeichnen.

Nach dieser Uebersicht der makroskopischen Verbältnisse gehe ich zur genauen Schilderung der mikroskopischen über. Ich beschränke mich für die Decidua auf eine Betrachtung der drei Seiten des Eies, auf welche es vollständig geschlossen ist. Die Dicke der Decidua schwankt zwischen $1 \frac{1}{2} \mathrm{~mm}$ bis 1 oder $\frac{1}{2} \mathrm{~mm}$, geht sogar hie und da etwas unter letztes Maass herunter. Am dicksten ist sie gerade an der angerissenen Seite und an derjenigen Längsseite, an der der intervillöse Raum am breitesten ist, also an der Stelle der späteren Placenta. Die äussere Begrenzung wird von einer ziemlich regelmässigen Linie gebildet, die im Grossen und Ganzen nicht den Charakter einer Rissfläche darbietet und nur an sehr wenigen Stellen finden sich einzelne schmale Balken, die den Zusammenhang mit der Uteruswand vermittelt zu haben scheinen und jetat durchrissen sind. Bei schwacher Vergrösserung sieht man sofort spaltförmige Oeffnungen (Gefässe), oft von $4-5 \mathrm{~mm}$ Länge, den beiden Seitenrändern des Bandes parallel laufend, und zwar dicht an seiner äusseren Fläche; sie finden sich namentlich da, wo die Decidua etwas dicker ist. Die Innenfläche ist leicht uneben und meist durch einen stark gefärbten Saum von etwas wechselnder Breite charakterisirt, nur an wenigen Stellen ist er auf eine ganz schmale rothe Linie beschränkt.

Diesem Saum liegen nach innen zu Zotten an, die meistens unter sich, wie (auch) mit der Decidua, durch eine stark rothe Substanz verbunden sind. Die genauere Zusammensetzung wird bei stärkerer Vergrösserung deutlicher.

Ich gehe hier von einer Stelle aus, wo die Zusammensetzung der Decidua am einfachsten ist und ibrer fötalen Fläche keine Zotten anlieges. Hier wird sie von grossen mächtigen Zellen gebildet, mit reichlichem, blassem, feinkörnigem Protoplasma, von einem deutlichen glänzenden, schmalen, hie und da doppelt contourirtén Saum (der Ausdruck einer Membran) umgeben. Der grosse ovale oder runde Kern ist hell gefärbt und enthält einige blasse Körnchen. Die Form der Zellen ist hie 
und da eine rundlich-polyedrische, doch weitaus die Mehrzahl ist in die Länge gestreckt, der Längsdurchmesser erreicht das Doppelte der Breite, die Enden sind abgerundet, die Zellform oval, oder sie Jaufen spitz zu, so dass die Zelle einer dicken, bauchig aufgetriebenen Spindel entspricht. Da diese längliche Zelle fast überall mit ihrer Längsaxe den beiden Seitenrändern des decidualen Bandes parallel laufen, so erhält dadurch die Decidua ein höchst charakteristisches längsstreifiges Aussehen. Ausser diesen Elementen finden sich Lymphkörperchen, oft in sehr bedeutender Zahl, und ferner noch eigenthümliche Kerne, die $z$ wischen den grossen Zellen als schmale und lange dunkle Stäbe eingeschaltet sind. Sie sind erheblich grösser, als die Kerne der Leukocyten und gehören also nicht Wanderzellen an. An die Enden dieser Kerne schliessen sich faserartige Ausläufer an, die sich hie und da noch ziemlich weit verfolgen lassen. An solchen Stellen erhält man den Eindruck, dass die grossen protoplasmareichen Zellen in den Maschen eines aus sternförmigen Zellen gebildeten Reticulums liegen. An manchen Stellen, namentlich nach der fötalen Fläche hin, sind die grossen Zellen noch mehr in die Länge gestreckt und schmaler, sie nehmen geradezu die Form einer langen Spindel an, deren Breite nur $\frac{1}{5}$ bis $\frac{1}{6}$ der Länge beträgt. Der rothe Grenzsaum nach dem intervillösen Raum hin besteht aus Fibrin, dessen Fasern oder Iamellen der Innenfläche parallel verlaufen; wo dasselbe etwas dicker ist, sieht man deutlich das gewöhnliche Netzwerk der Fibrinfasern. Eine sehr wechselnde Zahl von meistens einkernigen Lymphkörperchen ist darin eingeschlossen und bedeckt manchmal seine freie Oberfläche in grösserer Ausdehnung in fast continuirlicher ein- oder mehrfacher Lage. Andeutungen eines Saumes, welche einem Endothel (von mütterlichen Gefüssen) entsprechen könnten, fehlen.

Das Fibrin färbt sich sehr schön nach der Weigert'schen Methode (Färbung mit Gentianaviolett-Anilinwasser, Einwirkung von Jodjodkaliumlösung, Entfärbung mit Anilin-Xylol).

Von grosser Bedeutung ist das Verhalten der Drüsen. Durch die Untersuchungen, die gerade im letzten Jahre erschienen sind, ist der Nachweis geliefert, dass bei den Thieren, soweit sie untersucht sind, die Chorionzotten meist nicht in die 
Uterindrüsen hereinwachsen, wie man es sich früher lange Zeit vorstellte. Die Drüsen werden durch Wucherungen des Stromas der Schleimhaut verschlossen und sind daker für die Placentarbildung obne Interesse.

Wie verhält sich dies beim Menschen? Hier wird zur Zeit ziemlich allgemein angenommen, dass die Drüsen bei der Placentarbildung nicht in Betracht kommen. Man weiss, dass ihre tieferen Partien sich in weite, grosse Räume umwandeln, welche wesentlich die Hohlräume der sogenannten Drüsenschicht von Friedländer, der spongiösen und ampullären Schicht der späteren Autoren - bilden.

In der oberen compacten Lage der Decidua hat man an der reifen Placenta bis jetzt keine Drüsen gefunden. Dagegen sind sie in früheren Stadien noch vorhanden. Nitabuch findet in der von ihr untersuchten Placenta, aus dem Ende des fünften, oder aus dem Anfange des sechsten Monates, enge Drüsenöffnungen mit grossem Lumen und continuirlichem Cylinderepithel noch bis in die Höhe des Fibrinstreifens.

Minot ${ }^{1}$ ) lässt in seiner kürzlich erschienenen Arbeit „Ueber Uterus und Embryo" noch die Möglichkeit zu, dass eine in den grossen Placentarraum führende Oeffnung einer Drüse angehören könnte. Er sagt, es wäre kein Grund vorhanden, warum die Drüsenkanäle, welche fast vertical durch die compacte Lage der Decidua hindurchgehen, obliterirt sein sollten. Man könnte sogar dieselbe im Gegentheil in Folge von Dehnung des Uterus, erweitert erwarten. So könnte auch leicht Blut aus den intervillösen Räumen in die erweiterten Drüsen einfliessen.

K upffer (Münchener medic. Wochenschrift 1888. 515) fand an einem Ei aus dem Ende des ersten Monats, dessen Durchmesser $7 \frac{1}{2}$ und $11 \mathrm{~mm}$ betrugen, dass die Drüsen nicht in die Höhle der Fruchtkapsel einmündeten. Die in den Randpartien der Serotina zahlreichen Drüsen verlaufen sämmtlich in der Fläche dieser Membran gegen den Rand der Fruchtkapsel und münden in die Uterinhöhle derart, dass ein Kranz von Drüsenöffnungen die Basis der Reflexa umgiebt. Das Epithel dieser Drüsen war verändert, die Zellen stark vergrössert, cubisch, zum Theil kugelig aufgebläht und in Ablösung begriffen.

1) Journal of Jorphology. Vol. II. No. 3. April 1889. p. 426. 
Ich finde nun in dem vorliegenden Ei keine sichere Drüsenöffnungen in die grossen intervillösen Räume. Nur eine Drüse, welche senkrecht $\mathrm{d}$ ch die compacte Lage hindurchging, schien an einer Stelle, wo nur das oben beschriebene Grosszellgewebe, ohne Fibrinschicht, die Decidua bildete, in den intervillösen Raum sich zu öffnen. Indessen war dies in der Nähe der Rissstelle der mütterlichen Eihäute, so dass das Bild nicht beweisend sein kann. Alle übrigen Drüsen verhielten sich anders. Sie bildeten kürzere, meist spindelförmige Lücken von einem zur Oberfläche parallelem oder leicht schrägem Verlauf - an dem Cylinderepithel sind sie leicht zu erkennen; zwischen den helleren Cylinderzellen sind hie und da schmale dunkle Kerne eingeschoben; ich lasse es unentschieden, ob hier eine besondere Art Epithelzellen, oder Wanderzellen vorliegen. Verfolgt man eine solche Oeffinung auf einander folgenden Schnitten von unten nach oben, so sieht man, wie dieselbe der Fibrinschicht sich nähert, aber weit in der Mehrzahl erreicht sie dieselbe nicht. $\mathrm{Da}$, wo sie am nächsten an dieselbe herantritt, wird sie noch von zwei- bis dreifacher Lage von grossen Deciduazellen überlagert. Da hier vollständig continuirliche Schnittreihen vorliegen, so glaube ich dieses Resultat als feststehend ansehen zu dürfen. Weitaus die Mehrzahl sind also von dem grossen Placentarraum durch eine Wucherung des Stromas der Decidua geschieden. Nur zwei bis drei Lumina verhielten sich anders. Hier reichten die Drüsenöffnungen bis an das Fibrin, welches aber dieselben überbrückte und vollständig verschloss.

Ich schildere eine derselben ausführlicher (Fig. 2). Die betreffende Drüse bildet in ihrem obersten Theile eine etwas schräg zur Oberfläche ansteigende Spalte, durch 4 bis 5 Zellenlagen von der Fibrinschicht getrennt. An demjenigen Ende, welches der Oberfläche näher liegt, ist nun diese bedeckende Zelllage unterbrochen und der Drüsenkanal biegt fast rechtwinklig nach oben zu um. Die Stromagrenzlinie ist vollständig deutlich; sie ist nach dem Drüsenlumen hin etwas convex vorgebuchtet. Das hohe Cylinderepithel bricht an der Umbiegungsstelle scharf $a b$; der senkrecht nach oben emporsteigende Theil trägt kein Epithel, ist nur ein wenig schmaler, als der untere Theil, aber die Mündung ist nicht offen, sondern durch Fibrin verschlossen. Das- 
selbe senkt sich von oben her in den ausmündenden Kanal ein und erstreckt sich an der einen Wand bis an das Cylinderepithel heran. Seine Unterfläche verläuft schräg, und hier finden sich noch einige locker liegende Zellen, die, nach ihrer compacten Form, als Stromazellen anzusehen sind. Es sind dieselben im Begriffe die Drüsenmündung definitiv abzuschliessen, bilden aber noch keine zusammenhängende Lage. So sieht das Bild an dem mittleren von drei Schnitten aus. An dem ersten und dritten ist das Lumen etwas schmaler, das Fibrin füllt dasselbe bis an die Umbiegungsstelle vollständig aus und ist an seiner unteren Fläche von Cylinderepithel bedeckt.

Wenn wir die Oberfläche der Serotina betrachten, so finden wir, dass nur an recht beschränkten Stellen die oben beschriebene grosszellige Lage bis an den intervillösen Raum reicht. In der Regel liegt auf demselben die Fibrinschicht, und ferner noch auf dieser eine besondere zellreiche Schicht. Die letztere unterscheidet sich von den tieferen, schon bei schwacher Vergrösserung, leicht durch dichtere Lage der Kerne (Fig. 3).

Diese obere Schicht besteht aus rundlich-polyedrischen Zellen, ihre Grenzlinien stellen ganz feine blasse Linien dar, das Protoplasma ist sehr hell, der Kern ist rund, deutlich bläschenförmig, mit gefärbter Grenzlinie und mehr oder weniger Chromatinkörnern oder Fäden verseben. Die Zellen sind erheblich kleiner, als in der tieferen Schicht, ihre Kerne liegen viel näher - durchischnittlich nur um den eigenen Durchmesser von einander entfernt. Bei schwacher, wie bei starker Vergrösserung unterscheidet sich daher diese Schicht in sehr charakteristischer Weise von der tieferen. Die Kerne sind etwas stärker tingirt und treten um so deutlicher hervor, da die umgebende Zellsubstanz fast wasserklar ist. Die sehr feinen zarten Grenzlinien werden erst bei starker Vergrösserung sichtbar. Dies Gewebe bedeckt die Fibrinschicht in Form von Inseln, welche die verschiedensten Formen darbieten können. Bald füllt es kleine Unebenheiten derselben aus, wobei die Zellen manchmal schmal und dunkel, wie zusammengedrückt erscheinen und den seitlichen Flächen der Vertiefungen parallel gestellt sind, also senkrecht in die Tiefe verlaufen. Meist aber bildet es warzige Auflagerungen, flache Höcker, welche fünf, sechs und mehr Zellen in der Dicke, 
etwa 1-2 $\mathrm{mm}$ in der Länge messen, mit den Seitenflächen mehr oder weniger steil abfallen und nach der Zahl der Schnitte, auf denen sie sich wiederholen, ein ungefähr rundliches Feld der Serotina bedecken; oder dieses Gewebe liegt in grösserer Ausdehnung auf der Oberfläche der Fibrinschicht, so dass es auf einem Schnitt einen 4-5 mm langen Streifen bildet, aber im Ganzen von nur geringer Dicke, ein oder zwei Zelllagen enthaltend; an manchen Stellen erhebt es sich auch hier zu etwas dickeren, aber im Ganzen doch flachen Warzen.

Die Oberfläche dieses Gewebes nach dem intervillösen Raum ist also uneben; man kann wohl sagen, dass gerade die flachen Erhebungen und Höcker der Serotina, welche für die ersten Stadien von manchen Autoren beschrieben sind, an denen die Zotten sich inseriren, gerade von diesem zellreichen Gewebe gebildet werden. Mit dem eigentlichen Serotinalgewebe hat es noch das Gemeinsame, dass auch hier die sternförmigen, schmalen dunklen Zellen zwischen die hellen polyedrischen eingestreut sind, jedoch viel unregelmässiger, der Art, dass sie auch gar manchmal fehlen.

Nach dem intervillösen Raum ist dies Gewebe gar nicht selten noch durch einen schmalen, stärker gefärbten Saum begrenzt, der, wie starke Vergrösserung zeigt, Kerne enthält und aus abgeplatteten Zellen zu bestehen scheint. Ueber seine Bedeutung bin ich nicht in's Klare gekommen; entweder dürfte es sich um mütterliches Gefässendothel oder um Chorionepithel handeln.

Noch eine Eigenthümlichkeit habe ich zu erwähnen, die mir unverständlich geblieben ist. Manche dieser dickeren Warzen der wahrscheinlichen Placentarstelle sind von rundlichen und länglichen Oeffnungen durchbrochen, die von ganz regelmässigen Contouren begrenzt sind und Form und Grösse von Gefässöffnungen der darunter liegenden Serotina haben. Sie hängen unter einander zusammen und zeigen auch eine Ausmündung in den intervillösen Raum, so dass ich trotz des fehlenden Endothels immer nach einem Zusammenhang mit den darunter liegenden Gefässen suchte, aber immer vergeblich. Niemals konnte ich denselben finden, niemals durchbrach eine dieser Oeffnungen den darunter gelegenen Fibrinstreifen, um in das tiefere Serotinalgewebe einzudringen, 
Dies führt mich auf die Oeffnungen der mütterlichen Gefässe in den intervillösen Raum. Dies war eigentlich das Thema, von dem ich ausging. Aber die Oeffnungen waren offenbar nicht ganz normal, und namentlich gelang es mir nicht Arterien und Venen von einander zu unterscheiden. Abnorm war, dass sämmtliche sich öffnende Gefässe kein Endothel hatten.

Da für den Anfang des.6. Monats Nitabuch noch ganz regelmässig in den Venen Endothel fand, das nicht blos bis zur Mündung ging, sondern sich auch noch auf die Oberfläche der Serotina umschlug und dieselbe in weiter Ausdehnung bedeckte, so glaube ich vollen Grund zu haben, dies Fehlen im vorliegenden Ei als pathologisch ansehen zu dürfen. Und dem entsprechend waren denn auch sämmtliche dieser Gefässe thrombosirt, enthielten netzförmiges Fibrin, mit sehr zahlreichen farblosen Blutkörpern, das noch mit dem grosszelligen Gewebe der Wand fest verbunden war; zum Beweis, dass die Thrombose als vitaler Vorgang aufzufassen ist. Manche waren ganz damit angefüllt, andere nur zum Theil, der Thrombus fand sich vorzugsweise nur auf der einen Wand, die andere Hälfte des Lumens war fast frei. Die pathologische Bedeutung dieser Erscheinung für den Abort, sowie ihre etwaigen Ursachen will ich nicht besprechen; ich begnüge mich mit der Feststellung dieser Thatsache.

Was nun die Form und Anordnung der Gefässöffnungen anlangt, so kann ich mich kurz fassen, denn sie entsprachen der Schilderung, welche Nitabuch ${ }^{1}$ ) von den Venen giebt. Die langen, spaltförmigen Gefässe in der tieferen Serotinalschicht haben einen der Oberfläche fast parallelen oder fast schrägen Verlauf. An der Stelle der Oeffnung nähern sie sich der Oberfläche nur wenig und durchbrechen dieselbe. Die Begrenzung der Serotina nach dieser Oeffnung hin ist der Art regelmässig, dass der Gedanke an einen Riss ausgeschlossen ist. Dieses ausmündende Rohr ist nur sehr kurz, da die bedeckende Schicht der Serotina nur sehr dünn ist. Dagegen ist es weit, manch-

1) Die vorliegende Arbeit ist schon im November 1889 druckfertig gewesen. Ich habe daher noch die seitdem erschienene ausführliche Arbeit von Waldeyer (Archiv für mikr. Anatomie. 1890) anzuführen, deren Ergebnisse mit denen von Nitabucb übereinstimmen. 
mal sogar misst es 1-2 mm und mehr; - für ein Ei von dieser Grösse, wie mir scheint, eine Oeffnung von recht erheblicher Weite.

Solcher Oeffnungen sind nun recht viele, ich glaube 13 gefunden zu haben.

A ber wo sind die Mündungen der Arterien?

Einen sicheren Nachweis der letzteren könnte natürlich nur ihre Verfolgung bis zu dem noch mit Muscularis versehenen Gefäss liefern. Dieser Theil der Arterie war aber von vornherein nicht zu erwarten, da man sich nicht vorstellen kann, wie bei einem Abort die Durchtrennung eines Gefässes an seinem dickund festwandigen Theil erfolgen sollte. Wo ist die Gewalt, die dies besorgt? Die Lösung des Eies erfolgt nur in zellreichem, sehr weichem Gewebe.

Waldeyer, Nitabuch, Rohr und Bumm finden kurz vor der Arterienmündung dieselbe korkzieherförmig gekrümmt und diese Krümmungen in der Decidua gelegen. Die Durchschnitte dieses Arterienendes bilden eine Gruppe von rundlichen oder gebogenen Lumina, die sich in einander verfolgen lassen und immer einen runden Querschnitt haben. Solche Gruppen habe ich ebenfalls gefunden und ich habe natürlich auch vielfach versucht, die dazu gehörige Oeffnung festzustellen, aber meist vergeblich. Nur zweimal glaubte ich sie gefunden zu haben, aber das ausmündende Rohr verlief noch bevor es sich offnete, mehrere Nillimeter weit in der Serotina weiter and hatte auf diesem Verlauf, wie an der Mündung, so sehr das Aussehen der Venen, dass ich zu einer Entscheidung nicht kam.

Uebrigens hängen auch in die sich öffnenden Gefässe Zotten hinein und manchmal ziemlich weit, so dass sie auch an Schnitten sich fanden, an deuen das Gefäss nicht mit dem intervillösen Raum zusammenhängt.

Schliesslich noch einige Worte über den Inhalt des von der Decidua umschlossenen Raums: In vielen Schnitten liegt im Centrum das stark zusammengefaltete Amnion 0,018-0,02 mm dick, mit Epithel und der nach innen zu begrenzenden Lage von Zellen; dann das Chorion, etwas weniger, aber immer noch sehr stark gefáltet und collabirt, 0,5 mm dick, mit deutlich fibrillärem Stroma, reichlichen schmalen Spindelzellen und Gefässen an der 
Oberfläche. Das Stroma der Zotten hat die gleiche Zusammensetzung, doch ist es etwas lockerer, netzförmig gebaut, mit viel leeren, offenbar von Serum erfüllten Maschen.

Die von Langhans beschriebene Basalmembran ist hie und da als feiner doppelt contourirter Saum angedeutet, doch durchaus nicht überall.

Die Zellschicht ist an den Zotten überall ganz deutlich, die Zellen cubisch oder auch abgeplattet; am Chorion ist sie an vielen Stellen schwierig zu sehen, sie ist im Allgemeinen dünn, ihre Zellen sind also abgeplattet, aber immerhin sind die Stellen, wo man sich nicht mit Sicherheit von ihrer Existenz überzeugen kann, nur von sehr geringer Ausdehnung. Das Chorionepithel ist in gewöhnlicher Weise vorhanden, seine Dicke auch nur eine mässige, es ist mit zahlreichen Fortsätzen versehen.

Ferner finden sich nun zahlreiche grosszellige Inseln, an den Enden, zwischen den Zotten und selbst am Chorion von ganz demselben Aussehen, wie die grosszelligen Auflagerungen auf der Serotina; ganz dieselben polyedrischen Zellen mit den dicht stehenden Kernen, auch Lymphkörper und die scheinbaren Sternzellen finden sich hier, so dass ich auf ihre Zusammensetzung nicht weiter einzugehen brauche. Diese stehen in Zusammenhang: 1) mit den Zotten, wobei das Epithel auf die Aussenfläche der Inseln sich fortsetzen kann; die Zellschicht dagegen, die, wie gesagt, an den Zotten sehr dick ist, geht direct in das Gewebe der Inseln über; an diesem Zusammenhang ist kein Zweifel ; 2) mit der Serotina, d. h. mit deren gleich gebauten, über dem Fibrinstreifen gelegenen Schicht. Manchmal erscheint die Insel als Fortsetzung einer Zotte von gleicher Breite, die meisten aber sind breiter und dann können auch mehrere Zotten in sie meist unter Verlust des Epithels eintreten. Epitheliale Massen, rundliche, längliche, keulenförmige Ballen desselben finden sich auch in kleineren und grösseren Lücken der Inseln.

II.

Das Ei stammt aus der Privatpraxis des Herrn Privaldocenten Dr. Conrad. Frau B., 22 Jabre alt, ohne jegliche Erkrankung der Genitalien, aber an allgemeiner Anämie und Nervositäten leidend, hat noch niemals zeitig geboren, aber wahrscheinlich vor 6 Monaten abortirt. Die letzte Periode fand vor 5 Wochen statt. Erster kleiner Blatabgang vor 4 Tagen, stärkere 
Blutung und Austritt des Eies am 27. Februar 1886 Abends um 7 Uhr. Das Ei wird vor Eintrocknung geschützt, ohne Anwendung einer conservirenden Flüssigkeit aufgehoben und den 28. Februar Morgens, noch von ganz frischem Aussehen, Herrn Prof. Langhans übergeben. Nach äusserer Besichtigung und Eröffnung kommt es um 11 Uhr Morgens, 16 Stunden nacb der Ausstossung, in einprocentige Osmiumsäure für 24 Stunden, wird weitere 24 Stunden in fliessendem Wasser ausgewaschen und dann in $95^{\circ}$ Spiritus gelegt.

Das Ei bildete in frischem Zustande ein kleines ovales, Jeicht abgeplattetes Gebilde, von 15-18 mm Durchmesser, und erschien an seiner Oberfläche mit dicht stehenden kurzen Zöttchen besetzt, so dass scheinbar kein mütterliches Gewebe dasselbe umgab.

Das Ei schien vollständig frei zu liegen; beim Eröffnen entleerte sich eine etwas leicht trübe Flüssigkeit; ein Embryo fehlte. Ferner war noch èin grosses Stück Vera vorhanden. Das Eibläschen, in zwei Hälften zerschnitten, wird nach zwei Monaten in Celloidin eingebettet und in Schnitte zerlegt. Ohne jede Färbung war die Structur der einzelnen Gewebe sehr deutlich, nur das Chorionepithel war etwas dunkel, so dass seine Kerne häufig nur an besonders feinen Schnitten nacb der Färbung mit Häฒatoxylin sichtbar waren. Die Schnitte waren, wie es sich aus dem Angefübrten ergiebt, immer durch die eine ganze Hälfte des Eies geführt. Man hat an jedem Schnitte die geschlossene Eihöhle, umgeben von Chorion, dem nach aussen Zotten und eigenthümliche zapfenförmige Wucherungen der Zellschicht foigten, Amnion und Embryo fehlten.

Die Dimensionen der Eihöhle anzugeben ist überflüssig, da das Fi, in Folge der Härtung und Einbettung, etwas verzogen war. Es genügt die Angabe, dass die Wände der Eiböhle, d. h. Chorion sammt Zotten u. s. w., inrehschnittlich $1 \frac{1}{2} \mathrm{~mm}$ dick waren, mit geringen Scbwankungen; der Durchmesser der Eihöhle hat also in frischem Zustande etwa 12-15 $\mathrm{mm}$ betragen.

Nach dem makroskopischen Aussehen schienen Serotina und Reflexa zu fehlen, man musste daher auf der Aussenfläche des Chorion nichts, als Zotten erwarten. Seltsamer Weise war dagegen schon bei schwacher Vergrösserung nach aussen von den Zotten noch fast überall eine Lage von grosszelligem Gewebe zu sehen, die durch Zusammensetzung und Lagerung eine continuirliche Bekleidung des Eies mit Serotina und Reflexa vortäuschte (Fig. 4). Sie schien auf den ersten Blick continuirlich, aber bei genauerer Betrachtung sieht man leicht zahlreiche schmale Spalten, durch welche diese Schicht in zahlreiche kleine Stücke zerfällt. Die Stücke schliessen sich, wie die Steine eines Gewölbes zu einem zusammenhängenden vollständigen Ring an einander an. Die Aussenfläche dieses Ringes bot fast nirgends den Charakter einer Rissfläche dar, obgleich der Ring sich aus zahl- 
reichen kleinen Stücken zusammensetzte. Zwischen dieser anscheinenden Decidua und dem Chorion liegen nun die Zotten, nach dem Chorion hin mehr quer und schräg getroffen, etwas locker liegend, nach aussen dagegen von radiärem Verlaufe und sehr dicht gestellt, der Art, dass die schmalen Spalten zwischen ihnen immer feiner werden und schliesslich zum Theil in die feinen Spalten übergehen, welche den äusseren Ring durchsetzen. Sind die Zotten an einer Abgangsstelle getroffen (man sieht in jedem Schnitt 3-4 solche), dann laufen sie meist in gerader radiärer Richtung nach aussen, durchbrechen sogar den äusseren Ring, um in der Höhe seiner Aussenfläche zu enden. Manche Zotten sind kürzer und enden früher. Daraus ergiebt sich, dass die Zotten sofort nach ihrer ersten Verästelung meistens seitlich abbiegen, um erst in einiger Entfernung vom Chorion die directe Richtung nach dem mütterlichen Gewebe hin einzuschlagen. Nur eine kleine Zahl nimmt vom Anfang an den letzteren Verlauf. So ist das Bild bei Lupenvergrösserung. Aber schon bei hundertfacher Vergrösserung erkennt man, dass alle die länglichen, bandförmigen und runden Felder, die bisher als Längsund Querschnitte der Zotten bezeichnet wurden, durchaus nicht ganz von Zottenstroma eingenommen werden, sondern dass dieses und das peripherische grosszellige Gewebe sich fast zu gleichen Hälften darin theilen. Von dem peripherischen grosszelligen Ringe gehen also Fortsätze den Zotten entgegen, von gleicher äusserer Form, von gleicher Breite, wie diese und, wie wir sehen werden, auch von Epithel überzogen, so dass bei Lupenvergrösserung sie den Ziotten auf's Täuschendste gleich sehen. Ich bezeichne diese Fortsätze als Zellsäulen. Die Säulen und die Zotten theilen sich in das beschriebene Bild in etwas wechselnder Weise. Im Allgemeinen kommen sie sich auf halbem Wege entgegen; in anderen Fällen aber reichen die Säulen bis in die nächste Nähe des Chorions, aber ohne sich mit demselben zu verbinden. Ebenso reichen dann und wann auch die Zotten weiter nach aussen und manche unterbrechen selbst, wie erwähnt, den äusseren Ring, um aber genau in der Flucht seiner äusseren Fläche za enden.

Gehen wir nun zur näheren Betrachtung der einzelnen Bestandtheile des Eies über. 
Amnion, Nabelschnur, Dotterbläschen und Embryo fehlen. Auf der Innenfläche des Chorion liegt nur eine körnig-faserige Masse, in wechselnder Dicke, von undeutlichem Bau. Wir haben also, 1) Chorion, 2) Zotten und 3) peripherisches grosszelliges Gewebe.

Das Chorion bildet eine $0,1-0,12 \mathrm{~mm}$ dicke Lage von sebr blassem, mehr körnig, als fibrillär aussehendem Gewebe, mit spindel- und sternförmigen, durch Osmiumsäure geschwärzten Zellen, die der Oberfläche parallel oder leicht schräg zu ihr verlaufen und nur an den Abgangsstellen der Zotten senkrecht sich aufstellen, um in dieselben einzutreten. In den tieferen Schichten sind sie etwas grösser, wie in den oberen; sie hängen durch ihre Ausläufer zusammen. Die Blutgefässe charakterisiren sich als doppelte Reihen von Spindelzellen, so insbesondere in den mittleren und tieferen Schichten. Nach oben hin ist das Stroma fast überall durch einen feinen, doppelt contourirten Saum begrenzt, der geradlinig oder meistens wellenförmig verläuft, so dass in jeder Ausbuchtung eine Zelle der darüber gelegenen Zellschicht hineinpasst. In den Zotten ist das Stroma deutlich fibrillär, die Fibrillen von netzförmigem Verlaufe. Möglich, dass alle Fibrillen Ausläufer der sehr zahlreichen Zellen sind; Blutgefässe sind nicht $z u$ erkennen.

Die Zellschicht ist überall deutlich sowohl am Chorion, wie an den Zotten. Die Zellen sind hoch, sogar cylindrisch, ihr Protoplasma ganz hell. Der runde, meist ovale, grosse Kern, mit einigen wenigen Körnchen oder einem grossen Kernkörperchen versehen, in sonst hellem Inneren, liegt im oberen Theil der Zelle, bald quer, bald senkrecht gestellt. Die obere Fläche der Zelle ist meist stark convex, die dadurch entstehenden Vertiefungen sind von dem Epithel ausgefüllt, dessen dunkles Protoplasma auch häufig noch zwischen die Zellen bis an ihre Basis zu reichen scheint; die Zellen scheinen also in einzelne bienenwabenähnliche Nischen an der unteren Fläche des Epithels eingelassen. Ihre untere Fläche ist häufig noch stärker gewölbt und passt dann in die genannten Vertiefungen der Basalmembran. Das darüber liegende dunkle Epithel hat in seiner tieferen Schicht sehr zahlreiche Kerne, in der oberen, aber besonders an dem Chorion, sehr viele schwarz gefärbte Fetttröpfchen. Ich 
gebe zum Vergleich die Maasse der Kerne. Die runden Kerne des Epithels messen 0,005-0,006 mm, die der Zellschicht bis 0,008. Die ovalen sind im ersteren 0,004 breit und 0,008 lang, in der zweiten bis 0,006 breit und 0,01 lang.

Ich gehe nunmehr zu den Zellsäulen über. Ihre Zellen gleichen sehr denen der Zellschicht, sind aber etwas grösser; sie sind regelmässig polyedrisch, ohne dass eine Richtung besonders überwiegt; sie sind durch schmale glänzende Linien begrenzt (Ausdruck einer Membran), welche also alle in scharf ausgesprochenen rechten und stumpfen Winkeln zusammenstossen. Das Innere der Zellen ist sehr hell, man sieht in ihm eigentlich nur den Kern, der erheblichere Dimensionen erreichen kann, als wie in der Zellschicht (bis $0,012 \mathrm{~mm}$ in Breite und 0,02 in Länge). Nach aussen ändert sich die Beschaffenheit der Zellen insofern, als in ihnen ein homogenes, durch Osmiumsäure leicht graubraun gefärbtes Protoplasma auftritt. Hie und da verbreitern sich die Grenzlinien der Zellen zu einem etwas länglich dunkJem Knotenpunkte, der mit seinen 3-4 Ausläufern eine sternförmige Zelle nachahmt und auch nach Färbung mit Hämatoxylin schmale dunkle Kerne erkennen lässt. Diese Bildungen sind aber unbeständig und fehlen in einzelnen Säulen ganz, in anderen sind nur 2-3, in wieder anderen eine grössere Zahl, so dass man an ein Netz sternförmiger Zellen denken kann. Ihre Vertheilung ist sehr unregelmässig.

Dieses Gewebe bildet also Säulen, die in Form und Anordnung als Fortsätze der Zotten erscheinen, so dass an jede Zotte von radiärem Verlauf eine Säule sich anschliesst. Diese Säulen laufen nach der Peripherie und beginnen dann zu 2,3 oder 4 zu verschmelzen; auch die daraus entstehenden breiteren Säulen können sich wieder vereinigen und bilden so kleinere und grössere Stücke des bei schwacher Vergrösserung als zusammenhängend erscheinenden peripherischen Ringes von grosszelligem Gewebe. Die Länge solcher Stäcke beträgt bis $1 \mathrm{~mm}$ (der Durchmesser im Radius des Eies bis 0,2). So weit die Säulen getrennt sind, haben sie sehr häufig einen Ueberzug von Chorionepithel, das eine directe Fortsetzung des Zottenepithels ist. Nur an wenigen Zotten hört das Epithel gerade am Zottenende auf. Nach der Peripherie hin werden diese epithelialen Ueberzüge 
allmählich unterbrochen. Indess finden sich in den schmalen Spalten noch bis an den äussersten Rand hin kuglige und längliche Ballen des Epithels, von dem übrigen abgesondert, aber durch die Beschaffenheit des Protoplasmas und die Fetttröpfchen schon bei schwacher Vergrösserung leicht zu erkennen. Auch in den breiteren Säulen sind noch an vielen Stellen solche Epithelballen in etwas grösseren Lücken. Hie und da gehen von dem dunklen epithelialen Saume auch schmale Linien in die Säule hinein zwischen deren Zellen und scheinen mit den erwähnten dunklen Sternzellen zusammenzuhängen.

Was die intervillösen Räume anlangt, so sind dieselben, abgesehen von Epithelfortsätzen, die von den Ursprungsstellen abgeschnitten sind, leer. Sie enthalten keine Blutgefässe, auch nirgends eine Andeutung des wütterlichen Gefässendothels.

Die Decidua vera ist $\frac{3}{4}-1 \frac{1}{2} \mathrm{~mm}$ dick, hat eine sehr unebene Trennungsfläche, von welcher aus ihre korkzieherförmigen Drüsen nach oben in das Stroma hineingehen. Die obere Fläche ist uneben durch zahlreiche Drüsenöffnungen, die in Entfernung von nicht ganz $1 \mathrm{~mm}$ stehen. Die unteren drei Viertel der Vera haben eine Zusammensetzung, die derjenigen der Säulen ähnlich ist. Aber die Zellen sind grösser, ihr Kern misst 0,008-0,01, der Durchmesser der Zellen beträgt das Fünf- bis Sechsfache und mehr. Die Zellen sind rund oder polyedrisch, mit feinen Grenzlinien, hie und da sind dunkle längliche Kerne zwischen sie eingeschoben, ferner auch viele einkernige Lymphkörperchen; Grundsubstanz ist nicht deutlich.

Ganz anders ist die oberste Schicht der Vera zusammengesetat: hier sieht man viel helle Grundsubstanz und viel kleinere Zellen, deren Protoplasma grauschwarz gefärbt ist, deren Kern oval und gross ist, excentrisch gelegen. Der grösste Durchmesser der Zellen beträgt nur das Zwei- bis Dreifache des Kernes. Die Form der an der Oberfläche liegenden Zellen ist spindelund sternförmig, mit 3-6 Ausläufern, nach unten werden sie etwas grösser, nehmen runde compacte Form an und gehen so allmählich in die polyedrischen Zellen über.

\section{III.}

Das Ei stammt aus der Praxis des Herrn Privatdocenten Dr. Conrall. Es rührt von einer Frau ber, welche scbon mebrere ausgetragene Kinder ge- 
boren hat und in den letzten Jahren wiederbolt sebr frübzeitig abortirte, so am 9. December 1880 und am 9. Mai 1885. Die diesmalige Conception erfolgte entweder am 20. October oder am 9. November 1885. Nach mehrtägiger geringer Blutung wurde das vorliegende Ei am 9. December ausgestossen und sofort in feuchte Sublimatwatte gelegt, kam am 10. December in Müller'sche Flüssigkeit, 2 Tage nachher in Alkohol, am 16. December wird es in der Mitte durchschnitten, später in Celloidin eingebettet und mit dem Thoma'schen Mikrotom in Sehnitte zerlegt. Es entbielt keinen Embryo, auch Amnion und Nabelsebnur fehlten.

Das frische $\mathrm{Ei}$ stellt ein prall gefülltes kugliges Bläschen dar, von 15-18 $\mathrm{mm}$ Durchmesser und ist an der sichtbaren Fläche mit feinen Zöttchen bedeckt, welche es mit der Serotina und Reflexa verbinden. Leider löste sich diese Verbindung bei dem Wechsel der Erhärtungsflüssigkeiten; es wurde dabei eine kleine zottenfreie Stelle sichtbar, die der Reflexa angelegen hatte.

Die Abbildung (Fig. 6) stellt das Ei so dar, dass die Oberflächen von Vera und Reflexa nach unten liegen. Die Trennungsfläche der Vera mit wulstig polypösen Anhängen (erweiterte Drüsen) ist dem Beobachter zugekehrt, zu den Seiten des Eies erheben sich in rechtem Winkel zwei dünne Membranen, welche die durchrissene Serotina darstellen. Doch können dieselben über dem Ei nicht zum Schluss gebracht werden - ein kleiner Theil der Serotina fehlt offenbar.

Das Chorion ist $0,05 \mathrm{~mm}$ dick, sein Stroma fibrillär, und nach der körnigen der Innenfläche aufliegenden Masse (Rest des zu Grunde gegangenen Eiinhaltes) durch eine fast continuirliche Reihe von Spindelzellen begrenzt; gleiche Spindelzellen findeu sich dann in der Dicke des Stromas selbst mehr zerstreut. In den Zotten sind Stroma und Zellen sehr blass, manche Zotten sind sehr verbreitert und enthalten im Inneren eine grosse Höhle (beginnende Hydatidenmole); die Zellschicht ist am Chorion dünn, nicht überall deutlich und besteht aus schmalen Spindelzellen, die an den quer abgestutzten Enden sich verbinden. An den Zotten ist sie dick und leicht als continuirliche Lage zu erkennen; die basale Fläche der einzelnen Zellen ist stark convex und passt in entsprechende Ausbuchtungen des Stromas hinein. Das Epithel ist auch deutlich, mit ihm stehen hie und da kleine Fibrinheerde in Verbindung, auf deren Beschreibung ich nicht weiter eingehe. An den Enden der Zotten finden wir 
wieder Fortsätze von grosszelligem Gewebe. Ihre Gestalt wechselt, hie und da haben sie die Form der Zotten und bilden directe Fortsetzungen derselben, ganz so, wie bei dem eben beschriebenen Ei. Meist ist ihre Gestalt unregelmässig und mehrere Zotten treten an sie heran. Ihre Zellen haben deutliche Membranen, ein blasses feinkörniges Protoplasma, während die Elemente der Zellschicht ein wasserklares Innere haben -- ein Unterschied, der selbst da deutlich hervortritt, wo die Insel an den Enden einer Zotte liegt und in die Zellschicht übergeht. Zwischen ihnen finden sich hie und da auch sternförmige dunkel gefärbte Zellkörper und ferner Lymphkörperchen. In kleineren und grösseren Lücken finden sich Haufen von kernhaltigem Epithelprotoplasma.

Was nun Serotina und Reflexa anlangt, so wiederholen sich in deren Zusammensetzung alle Einzelheiten, die ich schon bei den ersten Eiern geschildert habe (Fig. 7). Ihr grosszelliges Gewebe ist nach der Eihöhle hin auch hier zunächst von einem Fibrinstreifen bedeckt und auf diesem liegt nun an den meisten Stellen, besonders reichlich an der Reflexa, wiederum grosszelliges Gewebe, die Zellen aber sind kleiner, wie die unter den Fibrinstreifen gelegenen. Dieses Gewebe bildet kleine warzenförmige Erhebungen oder bedeckt - in dünnerer Lage etwa von 2-3 Zellen in grosser Ausdehnung - die Innenfläche der mütterlichen Eihüute. Hie und da finden sich noch Reste des Chorionepithels, so dass die Aehnlichkeit mit den oben beschriebenen Inseln eine äusserst grosse ist.

An der Vera, von welcher ein grosses Stück mit abgegangen war, fehlte ein solches oberes grosszelliges Gewebe, sie besteht nur aus einem Gewebe, welches ganz dem Gewebe der tieferen Schicht von Serotina und Reflexa gleicht.

Ich fasse die wichtigeren Ergebnisse meiner Untersuchungen in einigen Sätzen zusammen.

1. Die Drüsen der Serotina münden nicht in den grossen Placentarraum, sondern sind gegen denselben durch eine 3 bis 4 Zellenlagen dicke Schicht der Serotina, seltener durch Fibrin, abgeschlossen. 
2. An der Serotina und Reflexa lassen sich folgende Schichten erkennen:

a) Eine tiefere, welche die Hauptmasse dieser Membranen bildet und wesentlich aus grossen protoplasmareichen, meist länglichen und der Oberfläche parallel gestellten Zellen besteht, mit Blutgefässen und Drüsen.

b) Diese wird nach dem Placentarraum von einer fast continuirlichen dünnen Lage von reticulärem, die charakteristische Farbenreaction Weigert's darbietenden Fibrin begrenzt.

c) Auf dieser liegt eine vielfach unterbrochene Schicht grosszelligen Gewebes, von verschiedener Dicke, von dem Fibrin oft noch durch lange schmale Spalten getrennt; seine Zellen sind kleiner als die der tieferen Schichte, polyedrisch, haben ein helles Protoplasma.

3. Das gleiche Gewebe findet sich in Form von Inseln zwischen den Zotten bis an das Chorion hin und geht in die Zellschicht der Chorionzotten über. Die Elemente der letzteren gleichen an den Uebergangsstellen völlig denen der Inseln.

4. Die Vera hat nur die Zusammensetzung wie die tiefere Schicht der Serotina. Die Fibrinschicht und das darauf liegende grosszellige Gewebe fehlt.

5. Alles dies spricht dafür, dass dieses - unter $2 \mathrm{c}$ und 3 beschriebene - Gewebe nicht aus dem Gewebe der Serotina hervorgeht, sondern fötalen Ursprungs ist.

6. Besonders interessant ist der Befund an Ei II. - Hier hat offenbar die Lostrennung des Eies gerade an der Grenzfläche der beiden Schichten stattgefunden. An dem ausgestossenen Ei ist also nur fötales Gewebe vorhanden.

Diese Resultate stimmen also wesentlich mit denen von Kastschenko überein, der jedoch die eigentlichen mütterlichen Eihäute nicht berührt hat.

Auf die Unterschiede seiner Anschauungen gegenüber den Ergebnissen der Langhans'schen Untersuchungen über die Ge. nese der Zellschicht gehe ich nicht weiter ein. Ich erwäbne nur, dass diese Schicht in meinen Präparaten continuirlich an Chorion und Zotten war. 
Es sei mir gestattet an dieser Stelle meinem hochverehrten Lehrer, Hern Professor Langhans, für seine liebenswürdige Hülfe meinen innigsten Dank auszusprechen.

\section{Erklärung der Abbildungen. Tafel IX.}

Fig. 1. Von Ei 1. 3/1. a Eiböhle, umgeben von dem gefalteten und daher ungleich dick erscheinenden Chorion. Die gleichmässig dunkel schattirten Flecke $z$ wischen den Zotten stellen die grosszelligen Inseln dar.

Fig. 2. Serotina mit Drüse von Ei I. 300/1. Beschreibung im Text.

Fig. 3. Serotina von Ei I. 300/1. Die beiden Schichten derselben durch den dunklen Fibrinstreifen getrennt.

Fig. 4. Ei II. Durchschnitt durch das ganze Ei. Chorion und Zotten sind hell gehalten. Inseln und Zellsäulen dunkel.

Fig. 5. Ei II. 100/1. Chorion, Zotten und Zellsiulen. Das Epithel ist nur durch eine dunkle Linie angedeutet. Die darunter liegende Reihe von Kernen gehört der Zellschicht an.

Fig. 6. Ei III. Erklärung im Text.

Fig. 7. Serotina von Ei III, mit Fibrinstreif und aufgelagerter grosszelliger Schicht. $100 / 1$. 